\title{
Orbit and optics distortion in fixed field alternating gradient muon accelerators
}

\author{
Shinji Machida* and David J. Kelliher \\ ASTeC, STFC Rutherford Appleton Laboratory, Chilton, Didcot, Oxfordshire, OX11 OQX, United Kingdom
}

(Received 21 May 2007; published 13 November 2007)

\begin{abstract}
In a linear nonscaling fixed field alternating gradient (FFAG) accelerator, betatron tunes vary over a wide range and a beam has to cross integer and half-integer tunes several times. Although it is plausible to say that integer and half-integer resonances are not harmful if the crossing speed is fast, no quantitative argument exists. With tracking simulation, we studied orbit and optics distortion due to alignment and magnet errors. It was found that the concept of integer and half-integer resonance crossing is irrelevant to explain beam behavior in a nonscaling FFAG when acceleration is fast and betatron tunes change quickly. In a muon FFAG accelerator, it takes 17 turns for acceleration and the betatron tunes change more than 10 , for example. Instead, the orbit and optics distortion is excited by random dipole and quadrupole kicks. The latter causes beam size growth because the beam starts tumbling in phase space, but not necessarily with emittance growth.
\end{abstract}

DOI: 10.1103/PhysRevSTAB.10.114001

PACS numbers: $29.20 .-\mathrm{c}, 41.75 .-\mathrm{i}$

\section{INTRODUCTION}

As a muon accelerator for a neutrino factory, a fixed field alternating gradient (FFAG) accelerator was proposed for its cost effective use of an rf system compared with other types of accelerators such as a linear accelerator and a recirculating linear accelerator [1]. An FFAG accelerator has a large acceptance and rapid acceleration possible. Although the first proposal adopted a so-called scaling type of FFAG as a candidate, recent studies show that a simplified lattice without the scaling law $[2,3]$ could be even more economical in terms of lattice magnets. This machine is called a nonscaling type of FFAG.

A nonscaling FFAG consists of only linear magnets, namely, dipole and quadrupole or combined function magnets [4-6]. It gives up the scaling law so that the betatron tune is not constant during acceleration. On the other hand, it squeezes the dispersion function so that the orbit shift between injection and extraction momenta can be small and therefore the magnet aperture is smaller than that for a scaling FFAG. This also makes the variation in revolution frequency from injection to extraction small so that the fixed rf frequency of around $200 \mathrm{MHz}$ can be employed.

One potential problem of a nonscaling FFAG is the tune excursion. Although the phase advance per cell, more specifically the number divided by $2 \pi$ or the cell tune, is adjusted between 0 and 0.5 so that there is no linear stability problem provided that all the cells comprising a ring are identical, the total tune of a ring varies over a few or even more than 10 units and crosses several integer and half-integer tunes. One may conjecture that such a crossing of integer and half-integer tunes would not be harmful if tune changes quite rapidly with energy. In fact, the 10 to $20 \mathrm{GeV}$ muon ring, for example, is supposed to finish

\footnotetext{
*s.machida@rl.ac.uk
}

acceleration in 17 turns. Within one turn, the total tune changes an order of 1 and a particle may not see periodicity of a ring. Nevertheless, without clear understanding of the effects of tune excursion over many integer and halfinteger tunes, it is hard to decide whether a nonscaling FFAG is the right choice for a muon accelerator.

There is an approach to overcome the tune excursion problem. A nonscaling FFAG with sextupole or higher order components, which is called a nonlinear nonscaling FFAG, may correct chromaticity at least partially and reduce the range of the tune excursion [7]. This might be essential if one would apply a nonscaling FFAG to slower cycling machines such as proton and carbon therapy accelerators. Studies to date, however, suggest that a nonlinear nonscaling FFAG is unable to meet the acceptance requirements needed for muon acceleration [8].

There exist some analytical studies on rapid betatron resonance crossing. Koscielniak and Baartman, for example, examine the growth of emittance, or precisely speaking, of the action variable, at an isolated resonance [9]. From a different point of view, Sturrock suggested that the effect of repeated rapid crossing might be approximated to a diffusion process [10]. In any event, we know that dipole errors introduce orbit distortion and quadrupole errors cause optical distortion in an alternating gradient accelerator. Numerical experiments with realistic accelerator and beam parameters and statistical analysis should give us quantitative as well as qualitative arguments.

The subject of this paper is to study how the orbit and optics distortion is excited and to estimate its magnitude with various error sources when the betatron tune moves quickly over many integer and half-integer numbers. After explaining the accelerator and beam model, we will look at orbit distortion due to alignment errors and quadrupole strength errors. We will then look at optical distortion due to quadrupole strength errors. We will discuss the 
random walk model as a source of distortion and simulation results are compared with the model. The findings will be summarized at the end.

\section{ACCELERATOR AND BEAM MODEL}

As an example of a linear nonscaling FFAG, we adopted the lattice and beam parameters of the 10 to $20 \mathrm{GeV} / c$ muon accelerator proposed in a neutrino factory design study [11]. The circumference is about $410 \mathrm{~m}$ and the whole lattice consists of 84 identical cells. Each cell has focusing and defocusing quadrupoles that make a doublet focusing structure. The excursion of the cell tune is shown in Fig. 1. Both quadrupoles are shifted in radial direction by different amounts so that a beam going through the quadrupoles receives bending action like in a combined function magnet. We do not assume that there were independent knobs for dipole and quadrupole strength. Dipole kick is made by a feed down from the shifted quadrupole magnet. Although the lattice with shifted quadrupoles is not the only way to realize a linear nonscaling FFAG, it is at least feasible from an engineering point of view. In practice, however, it should be noted that alignment errors of the quadrupoles introduce both horizontal and vertical orbit distortion. The quadrupole strength errors introduce horizontal orbit distortion as well as horizontal and vertical optical distortion.

A muon beam is accelerated from 10 to $20 \mathrm{GeV} / c$ in 17 turns. The total turn number can be adjusted by rf voltage. Since the acceleration time is so short, the rf frequency cannot be synchronized with beam revolution frequency. Therefore, the so-called "gutter acceleration" scheme is

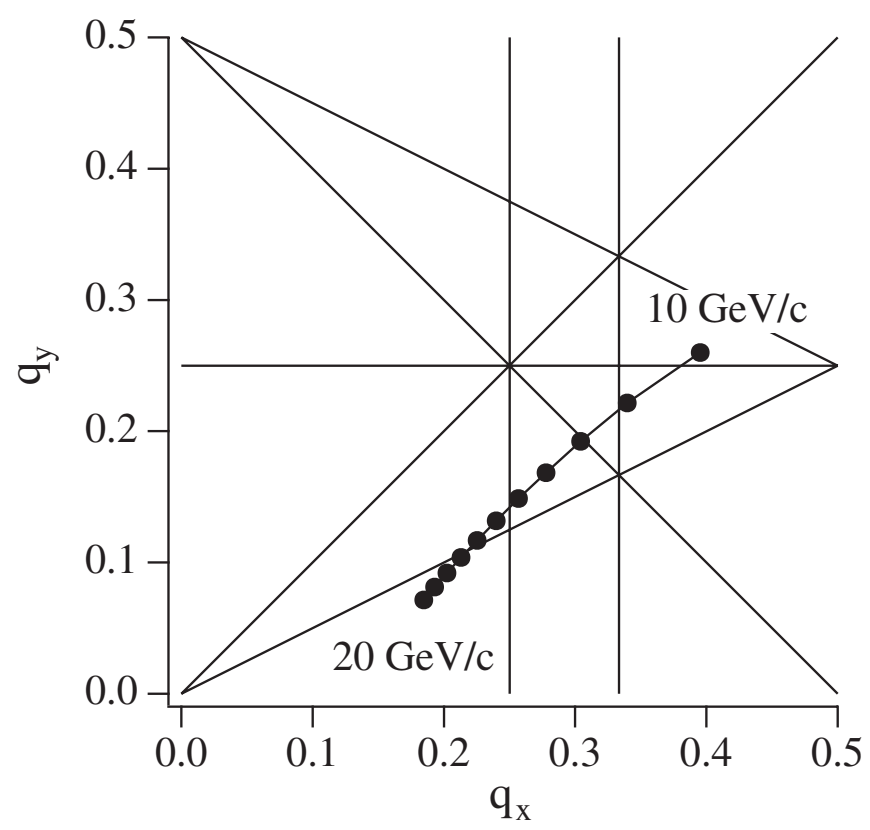

FIG. 1. Excursion of the cell tune from injection to extraction. Filled circles are marked every $1 \mathrm{GeV} / c$. Structure resonance lines up to fourth order are also shown. employed which uses a flow outside an rf bucket of a fixed frequency in longitudinal phase space [12,13]. Lattice parameters are chosen such that the path length depends on momentum quadratically. In this way, the radial orbit excursion can be reduced as well. The variation of revolution frequency becomes minimum because the muon is already relativistic at injection momentum.

Although there is a phase shift with respect to the rf crest during acceleration, we assumed that the beam gains constant energy every time it goes through an rf cavity. That can be justified by small phase shift when a beam is accelerated quickly in 17 turns. There was another reason we had to take this model. That is the revolution frequency variation depending on transverse amplitude. As we have discussed in another paper, path length depends on transverse amplitude in a nonscaling FFAG since it is operated with natural chromaticity [14]. The normalized muon beam emittance assumed of $30 \pi \mathrm{mm}$ rad gives a significant phase shift and some particles will be out of acceleration phase. Although this has to be remedied by partial chromaticity correction [15], etc., a study of transverse dynamics that we are going to discuss should be free from detailed longitudinal motions except rapid change of momentum and betatron tune.

A nonscaling FFAG does not have dispersion free sections and rf cavities have to be located at a place with a finite dispersion function. Although the magnitude of dispersion is small compared with an ordinary synchrotron, the energy gain per cavity is large: about $10 \mathrm{MeV}$, and sudden jump of the equilibrium orbit becomes a concern. We have looked at the orbit distortion as a function of the number of rf cavities. There is no error in the lattice.

Figure 2 shows the horizontal orbit distortion when there is an rf cavity every 2 and 3 cells, respectively. Since the total number of cells is 84 , there exists symmetry of 42 and 28 . The total voltage per turn was kept constant. Abscissa is the horizontal cell tune and it has a larger value at the left side since the tune is higher at injection and decreases as it is accelerated. When there is an rf cavity every 2 cells, almost no orbit distortion appears. With a fewer number of rf cavities, however, orbit distortion is excited at a particular cell tune during acceleration. It is around 0.33 with every 3 cells and around 0.25 with every 4 cells. With every 14 cells, there are three steps around $0.35,0.28$, and 0.21 .

This is a resonance excited locally within a small periodic structure introduced by rf cavities. A sudden jump of the equilibrium orbit with an rf cavity every $n$ cells becomes in phase when the cell tune $q x$ is $n q x=m$, where $m$ is an integer. In the range of horizontal tune of 0.40 and 0.18 , there is one cell tune which satisfies a resonance with an rf cavity every 3 and 4 cells and three tunes which satisfy resonances with an rf cavity every 14 cells. We may say that is a synchrobeta coupling resonance although the synchrotron tune is zero. In the following study, the lattice always has an rf cavity every 2 cells to avoid the resonance. 

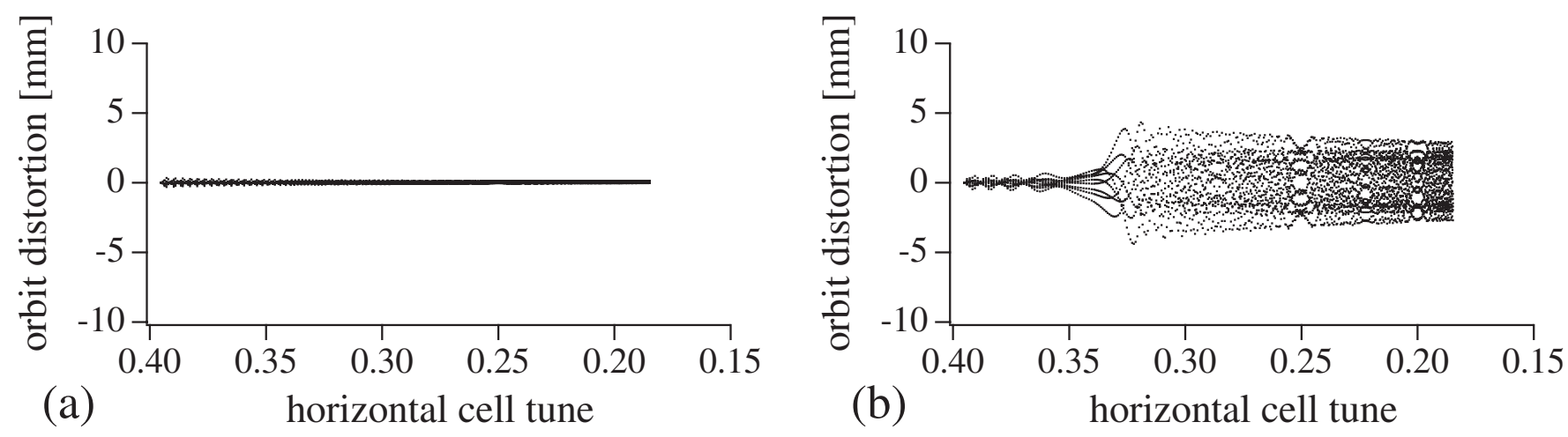

FIG. 2. Horizontal orbit distortion with different number of rf cavities. rf cavities are installed every (a) 2 and (b) 3 cells.

\section{ORBIT DISTORTION}

\section{A. Definition}

We first looked at the orbit distortion throughout acceleration. Unlike an ordinary synchrotron and a scaling FFAG, the phase advance per cell changes as the beam is accelerated. That makes betatron tune cross many integers and half-integers as we have already mentioned. In other words, orbit distortion changes dynamically. By tracking a particle with acceleration, we investigated orbit distortion in two cases: with alignment errors and with gradient errors.

In order to estimate the orbit distortion in practice, horizontal and vertical positions of a particle that was tracked to extraction momentum were recorded at each magnet and at an rf cavity without any errors. Strictly speaking, each magnet in tracking is represented as a group of thin elements. Horizontal and vertical positions are recorded at each thin element. The difference in the horizontal closed orbit from injection to extraction momentum is around $60 \mathrm{~mm}$ at the center of a straight section. The vertical closed orbit is always zero without errors. The initial position and gradient were chosen at the closed orbit defined at injection momentum. After introducing errors, a particle was tracked again. This time, the initial position and gradient were chosen at the new closed orbit which was calculated including the errors. The difference between two trajectories was defined as orbit distortion. When an alignment error was included, two magnets that comprise of doublet focusing were misaligned in the same direction and amplitude because both may sit on the same support table in reality.

\section{B. Alignment errors}

In order to estimate the magnitude of orbit distortion, we introduced 501 patterns of alignment errors to a single lattice, which give 501 different patterns of orbit distortion. We calculated the maximum distortion produced by each pattern and plotted as a function of the magnitude of misalignment as shown in Fig. 3. In order to quantify the results, plots of the maximum data were fitted by a linear function. We then introduced "amplification factor" as a coefficient of a linear part,

amplification factor $=\langle$ orbit distortion $[\mathrm{mm}] /$ misalignment $[\mathrm{mm}]\rangle$

where \langle\rangle denotes taking the average over all patterns. The amplification factor for horizontal and vertical directions becomes 143 and 110, respectively. Namely, a misalignment of $0.1 \mathrm{~mm}(\mathrm{rms})$ likely excites the maximum orbit distortion of $14 \mathrm{~mm}$ in the horizontal and $11 \mathrm{~mm}$ in the vertical plane.

\section{Gradient errors}

Since we assume that dipole fields are created by the shifted quadrupoles, horizontal orbit distortion can be also excited by gradient errors. Similar to alignment errors, we introduced 501 patterns of gradient errors to a single lattice, which give 501 different patterns of orbit distortion. As a function of gradient errors, maximum orbit distortion

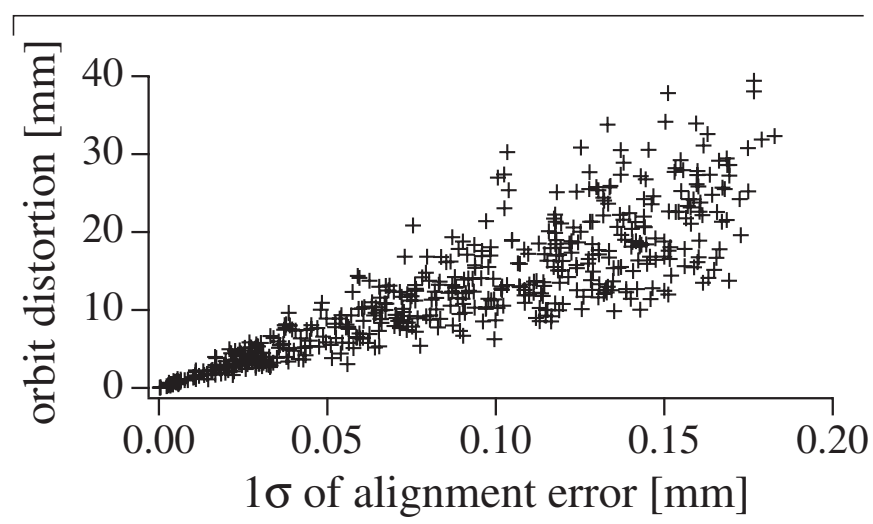

FIG. 3. Distribution of maximum horizontal orbit distortion out of 501 different alignment errors. 


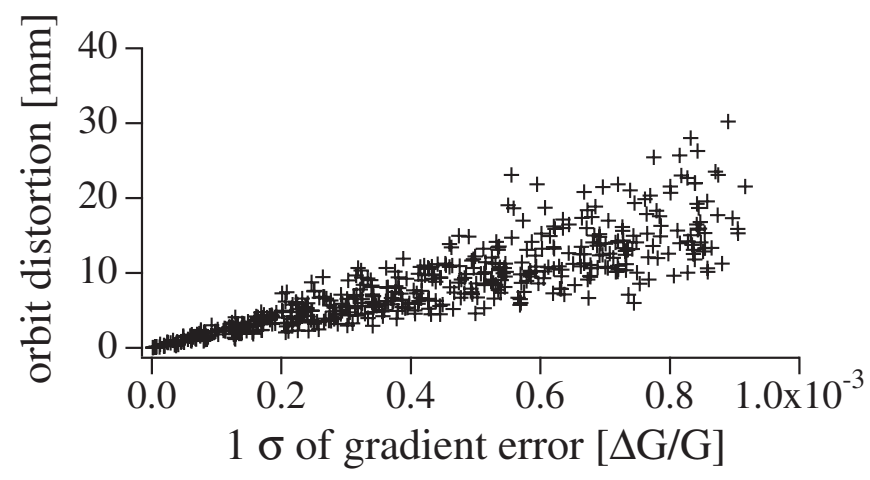

FIG. 4. Distribution of maximum horizontal orbit distortion out of 501 different gradient errors.

of 501 different cases is plotted in Fig. 4, which gives linear increase of distortion as expected.

Quantitatively, the magnitude of distortion is reasonable taking into account the following numbers. Since a particle goes off-axis at about $0.18 \mathrm{~m}$ in defocusing quadrupole (QD) and the gradient of QD is about $29 \mathrm{~T} / \mathrm{m}$, the gradient error of $1 \times 10^{-3}$ corresponds to an alignment error of $0.18[\mathrm{~mm}]$. Namely, the maximum orbit distortion of around $20 \mathrm{~mm}$ at the gradient error of $1 \times 10^{-3}$ is consistent with the distortion at the alignment error of $0.18[\mathrm{~mm}]$. We did not consider the bending action due to focusing

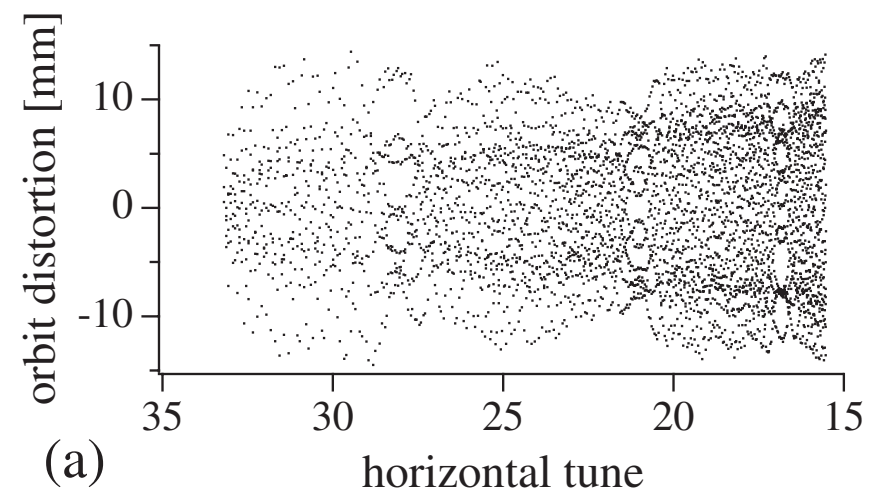

quadrupole $(\mathrm{QF})$ because the offset of the axis is $0.045 \mathrm{~m}$ and the gradient is $24 \mathrm{~T} / \mathrm{m}$. Clearly, the gradient error of $\mathrm{QF}$ does not contribute much to the orbit distortion.

\section{Integer stopband integrals}

One question is whether the orbit distortion is a result of integer tune crossing and resonance excitation. Figure 5 shows an example of horizontal and vertical orbit distortion of one pattern. We plot them as a function of the tune instead of time or momentum. Those figures should be compared with the integer stopband integrals of Eq. (1) [16] at each integer assuming the same errors:

$$
f_{k}=\frac{1}{2 \pi Q} \oint \sqrt{\beta} \frac{\Delta B}{B \rho} \exp (-j k \phi) d s
$$

and

$$
\phi(s)=\frac{1}{Q} \int_{0}^{s} \frac{d t}{\beta(t)},
$$

where $Q$ is tune, $\beta$ is a lattice function, $\Delta B$ is dipole kick due to alignment error, $B \rho$ is rigidity, and $k$ is integer.

As shown in Fig. 6, where the maximum stopband integral is normalized to one, there is no clear correlation between orbit distortion and integer stopband integrals. In this particular example, $1 \sigma$ of alignment error is around

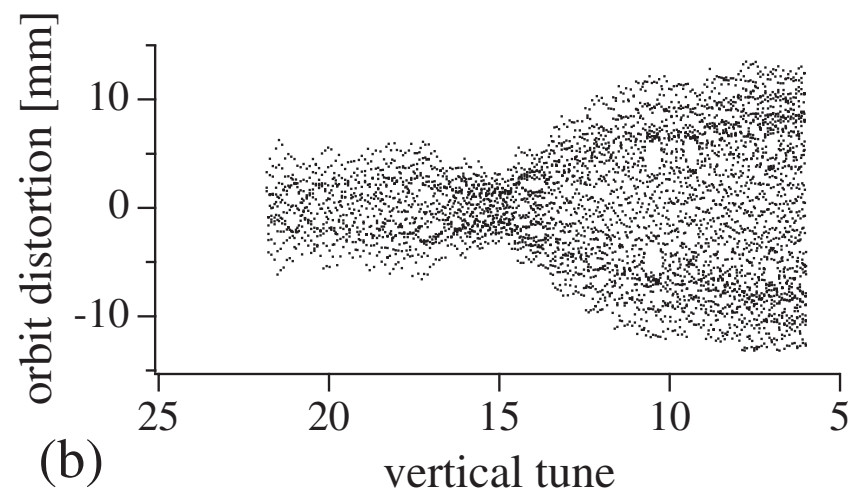

FIG. 5. Evolution of (a) horizontal and (b) vertical orbit distortion when alignment error of $0.10 \mathrm{~mm}(1 \sigma)$ is included.
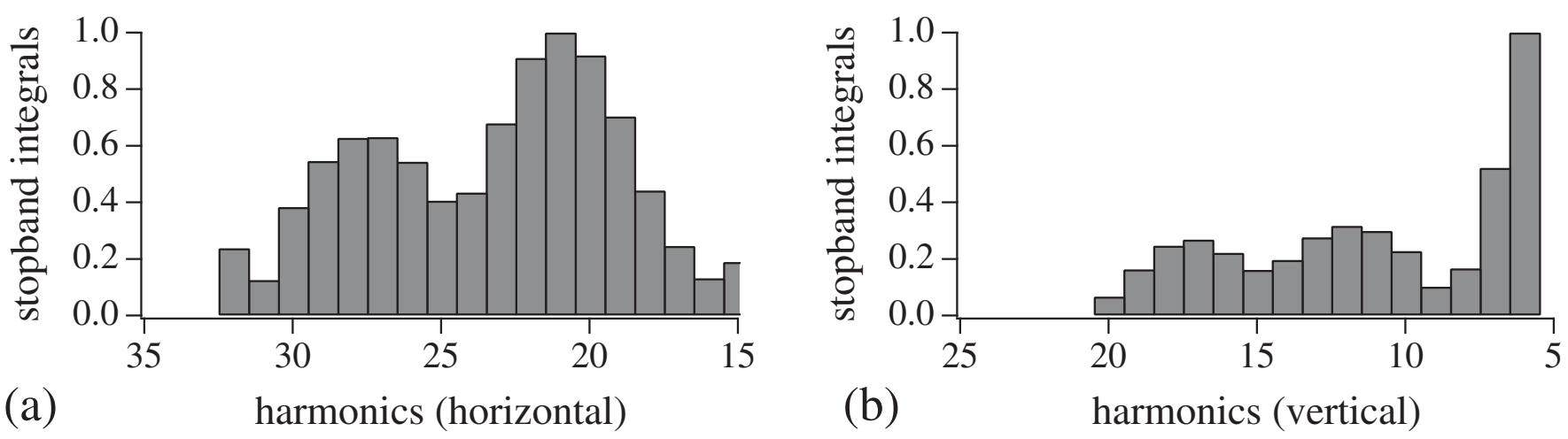

FIG. 6. (a) Horizontal and (b) vertical stopband integrals of a lattice with the same alignment errors as Fig. 5. 
$0.1 \mathrm{~mm}$ and the distribution is Gaussian. There is a cutoff at $2 \sigma$.

\section{OPTICS DISTORTION}

\section{A. Definition}

Once gradient errors are included in the lattice, optics is distorted or half-integer resonance may be observed. While the orbit distortion is clearly represented by the deviation from the ideal orbit, the optics distortion could be a more vague concept and should be defined in a rigorous way. To quantify the distortion, let us introduce the following parameters. First, we tracked 36 macroparticles on the edge of a $0.003 \pi \mathrm{mm} \mathrm{rad}$ emittance ellipse without gradient errors. All the particles had the same initial momentum and they gained the same energy at cavities so that the momentum spread was always zero. In this study, we only looked at the horizontal motion.

At the center of the long straight section, we calculated

$$
\begin{gathered}
\beta_{x}=\frac{\left\langle x_{i}^{2}\right\rangle}{\varepsilon_{x, \mathrm{rms}}} \\
\alpha_{x}=-\frac{\left\langle x_{i} p_{x, i}\right\rangle}{\varepsilon_{x, \mathrm{rms}}},
\end{gathered}
$$

where $\varepsilon_{x, \mathrm{rms}}=\sqrt{\left\langle x_{i}^{2}\right\rangle\left\langle p_{x, i}^{2}\right\rangle-\left\langle x_{i} p_{x, i}\right\rangle^{2}}$ is rms emittance, $x_{i}$ is horizontal position, $p_{x, i}$ is horizontal momentum, and \langle\rangle is the average over 36 particles. Remember that the phase advance and the lattice functions vary as a function of momentum. Figure 7 shows $\beta$ and $\alpha$ as a function of the horizontal tune. We named them as the fixed $\beta$ and the fixed $\alpha$ functions.

With the fixed $\beta$ and $\alpha$ functions, the amplitude of each macroparticle could be calculated with

$$
A_{i}=\frac{x_{i}^{2}+\left(\beta_{x} p_{x, i}+\alpha_{x} x_{i}\right)^{2}}{\beta_{x}} .
$$

Without any errors, the amplitude of all the macroparticles should be the same and constant during acceleration. Once

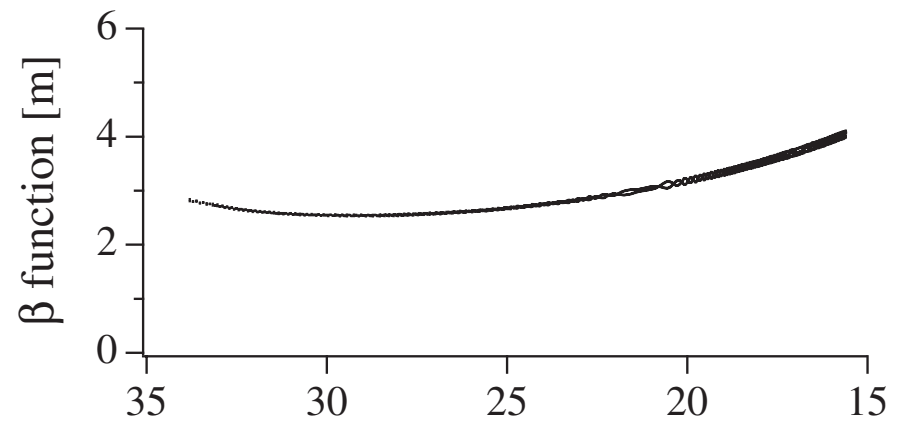

(a) horizontal tune gradient errors were included and optics was distorted, however, the amplitude defined above varies. We define the optics distortion as a square root of the maximum $A_{i}$ out of 36 particles normalized by a square root of the initial amplitude $A_{0}$, namely $\sqrt{\frac{A_{\max }}{A_{0}}}-1 \approx \frac{1}{2} \frac{\Delta A_{\max }}{A_{0}}$. We call $\frac{\Delta A_{\max }}{A_{0}}$ amplitude growth.

If the optics distortion is purely caused by gradient error and the optics remains linear, a proper choice of the $\beta$ and $\alpha$ functions give the constant amplitude for all the particles even though the distortion is significant. Those $\beta$ and $\alpha$ functions can be found by calculating Eqs. (3) and (4) in the same way, but for each individual lattice with errors. We named them as the fitted $\beta$ and fitted $\alpha$ functions. The amplitude calculated by the fitted $\beta$ and $\alpha$ functions tells us the real emittance increase if any while the optics distortion calculated by the fixed $\beta$ and $\alpha$ functions indicates beam size growth due to tumbling.

\section{B. Gradient errors}

As we did for the orbit distortion study, 501 patterns of gradient errors applied to a single lattice were prepared for statistical arguments. Then, the maximum amplitude out of the 36 macroparticles calculated both with the fixed and the fitted lattice functions was plotted in Fig. 8 as a function of gradient errors. This clearly shows that the amplitude defined with the fitted lattice functions stays constant, namely, the optics remains linear and emittance does not change at all, while the one with the fixed lattice functions grow more or less linearly. In the phase space, a beam tumbling occurs which results in beam size growth. In order to quantify the results, data were fitted by a linear function. We then introduced "growth factor" as a coefficient of a linear part,

growth factor $=\langle$ amplitude growth $/$ gradient error $\rangle$,

where \langle\rangle denotes taking the average. Growth factor for the fixed lattice functions is 250 and one for the fitted lattice functions is 0 . Namely, $1 \times 10^{-3}$ gradient errors (rms)

FIG. 7. Evolution of lattice functions (a) $\beta$ and (b) $\alpha$ in an error-free lattice. 


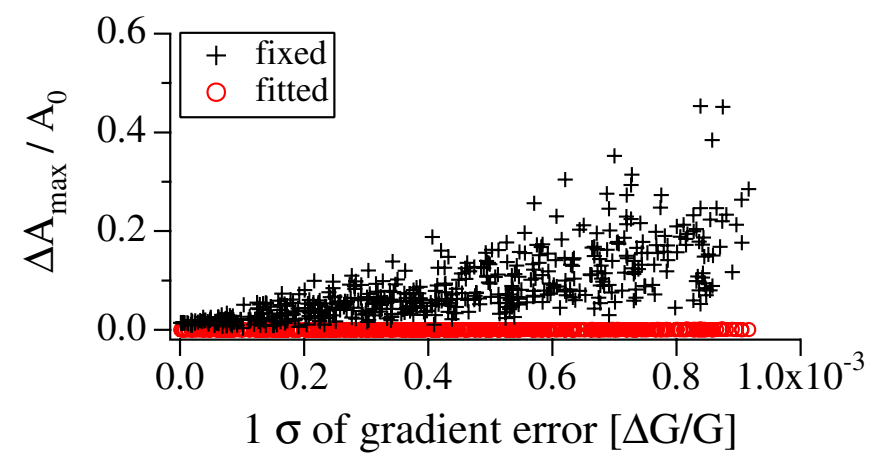

FIG. 8. (Color) Distribution of maximum distortion defined by the fixed and fitted lattice functions out of 501 different gradient errors. Initial emittance is $0.003 \pi \mathrm{mm} \mathrm{rad}$.

gives $12 \%\left(=\sqrt{1+250 \times 10^{-3}}-1\right)$ beam size growth but there is no real emittance increase.

\section{Emittance dependence}

So far, small emittance was assumed because the optics distortion is in principle independent of emittance. However, a muon beam is huge, namely $30 \pi \mathrm{mm} \mathrm{rad}$, and it is interesting to see if the optics distortion is independent of emittance to that level. Distributions of the amplitude with the fixed and fitted lattice functions show no difference between the initial emittance of $0.003 \pi \mathrm{mm} \mathrm{rad}$ and $0.3 \pi \mathrm{mm}$ rad. Figure 9 shows, however, a huge growth factor when the initial emittance is $30 \pi \mathrm{mm} \mathrm{rad}$. In this case, the amplitude with the fitted functions is no longer constant. This is because nonlinear distortion starts becoming significant in a huge emittance beam. This is due to a real increase of emittance.

Possible nonlinear sources are in the fringe field or in kinematic terms. Although it is not possible to switch off kinematic terms in our simulation code, we can see how the behavior changes when we model the fringe field in different ways. So far, we assumed the Enge falloff in the frame of the scalar potential in the cylindrical coordinate system

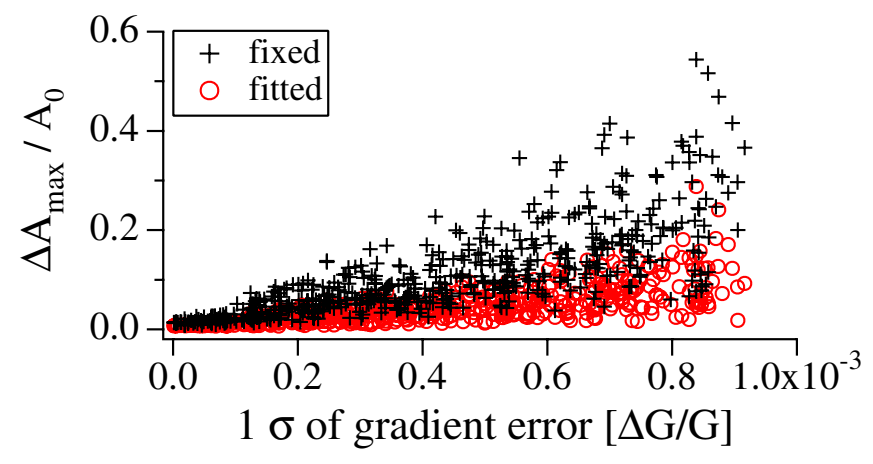

FIG. 9. (Color) Distribution of maximum distortion defined by the fixed and fitted lattice functions out of 501 different gradient errors. Initial emittance is $30 \pi \mathrm{mm} \mathrm{rad}$.

$$
P_{2}(r, \theta, z)=\frac{r^{2} \sin 2 \theta}{2}\left[G_{2,0}(z)+G_{2,2}(z) r^{2}+\cdots\right],
$$

where

$$
G_{2,2 k}(z)=(-1)^{k} \frac{2}{4^{k} k !(2+k) !} \frac{d^{2 k} G_{2,0}(z)}{d z^{2 k}}
$$

and

$$
G_{2,0}(z)=\frac{G_{0}}{1+\exp \left(\sum_{i=0}^{5} C_{i} z^{i}\right)}
$$

where

$$
z^{\prime}=\frac{z}{g}
$$

$C_{i}$ are the Enge coefficients, and they are $c_{0}=0.1455$, $c_{1}=2.267, c_{2}=-0.6395, c_{3}=1.1558, c_{4}=c_{5}=0$ in this study. $g$ is the order of the aperture and it is $0.1 \mathrm{~m}$.

The hard edge model, which models the vertical focusing as a delta-function kick, instead of the Enge falloff as the fringe fields gives slightly smaller growth factor: 270 (hard edge) vs 280 (Enge) with the fixed lattice functions and 92 (hard edge) vs 107 (Enge) with the fitted lattice functions. The major nonlinear source seems to be kinematic terms although there is some contribution from the fringe fields.

\section{Half-integer stopband integrals}

Again, a question is whether the optics distortion is a result of half-integer tune crossing and resonance excitation. Figure 10 is one example of amplitude growth during the acceleration and it should be compared with the halfinteger stopband integrals of Eq. (10) [17] at each halfinteger. The maximum stopband integral is normalized to one in Fig. 11:

$$
J_{p}=\frac{1}{2 \pi} \oint \beta \Delta k(s) \exp (-j p \phi) d s
$$

and

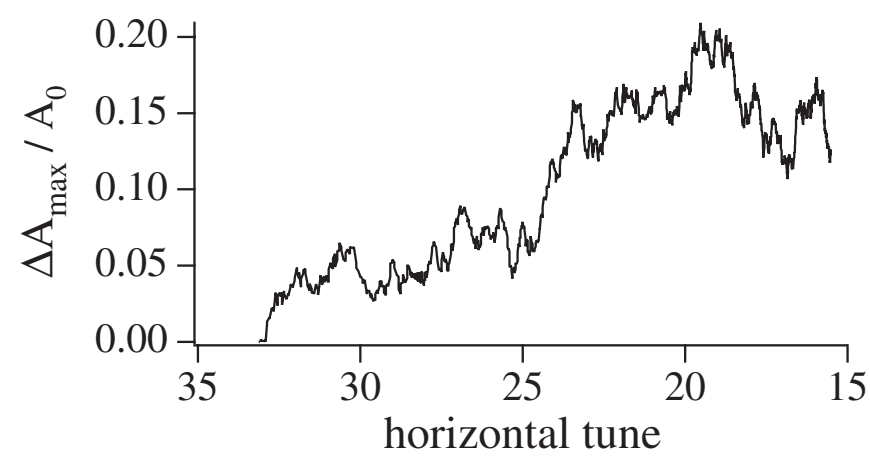

FIG. 10. Evolution of horizontal optics distortion when gradient error of $0.1 \%(1 \sigma)$ is included. 


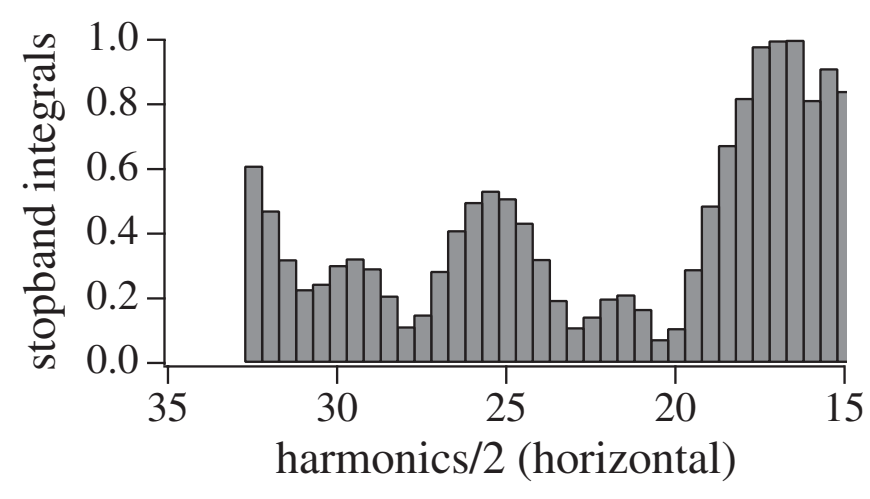

FIG. 11. Horizontal stopband integrals of a lattice with the same gradient errors as Fig. 10.

$$
\phi(s)=\frac{1}{Q} \int_{0}^{s} \frac{d t}{\beta(t)},
$$

where $\Delta k$ is quadrupole kick due to gradient error, and $p$ is integer.

There is no clear correlation between the distortion of lattice functions and half-integer stopband integrals. In this particular example, $1 \sigma$ of gradient error is around $1 \times$ $10^{-3}(=\Delta G / G)$ and the distribution is Gaussian. There is a cutoff at $2 \sigma$.

\section{DISCUSSIONS}

\section{A. Random walk model}

Tracking results show that orbit and optics distortion do not have any clear dependence on the betatron tune. It seems to be caused rather by random dipole and quadrupole kicks due to alignment and gradient errors. The reason we said random is the fact that phase advance per cell changes as a particle is accelerated and therefore the kicks due to errors are not periodic in phase space even though

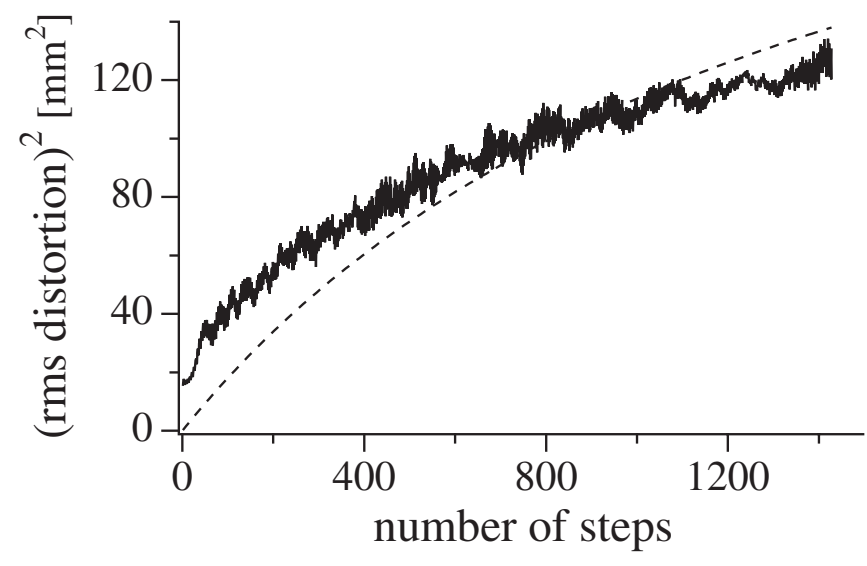

FIG. 12. Evolution of the square of rms orbit distortion. There are $1428(=84$ cells $\times 17$ turns $)$ steps. The solid curve is simulation results and the dashed curve is the random walk model. the magnetic fields are constant and physical locations of the kicks are fixed in a ring.

If the kicks are completely random and the rms strength of the kicks is constant, rms orbit distortion calculated at each time step over lattices with different alignment error patterns should give a square root dependence as a function of time. That is called the random walk model. Therefore, the time dependence of the distortion should give us the idea of the distortion source.

In our case, the rms strength of the kicks is not constant because the beam momentum increases and eventually becomes twice as much due to acceleration. The kick angle reduced as $\frac{1}{1+t}$ even though the absolute strength of the dipole kicks are constant, where $t=0$ at injection and linearly increases to 1 at extraction. In this case, the rms growth is proportional to $\sqrt{\frac{t}{1+t}}$ instead of $\sqrt{t}$ (see Appendix).

We have calculated rms orbit distortion over 501 patterns with fixed rms alignment errors of $0.1 \mathrm{~mm}$. Figure 12 shows the squared value of the rms distortion over 501 patterns and the expected curve by the model. The two curves agree reasonably well. Although one example of a pattern did not show any increase as in Fig. 5, statistical behavior reveals the nature of the kicks. The difference can be attributed to other factors such as the shift of orbit during acceleration which changes the absolute strength of dipole kicks as well. The correction due to the shift is unfortunately not as easy as the momentum increase.

\section{B. Dependence on acceleration rate}

Another way of confirming the source of distortion, which we assume is due to random kicks, is to calculate the distortion with different acceleration rates. Even though the details of time dependence of the kick magnitude is unknown, the final magnitude of distortion should have a square root dependence on time because the shape of Fig. 12 should be the same with different acceleration rate. Different acceleration rate could be simulated simply by higher or lower voltage at rf cavities. (Remember our model of acceleration is independent of $\mathrm{rf}$ phase and the energy gain is constant.)

Figure 13 shows the squared value of the rms distortion at the end as a function of total turn number which is required to finish the whole acceleration. Up to 51 turns, which is 3 times slower than the nominal acceleration rate, the square of the distortion linearly depends on the total turn number as expected. Distortion with even slower rate, however, starts deviating from the linear dependence.

We found that the exchange of orbit distortion between horizontal and vertical planes appears when the acceleration rate is more than 3 times slower than the nominal value as shown in Fig. 14. That accounts for the deviation from the linear dependence. From the timing of the exchange, $q x-2 q y=0$ resonance is identified as the source of the 


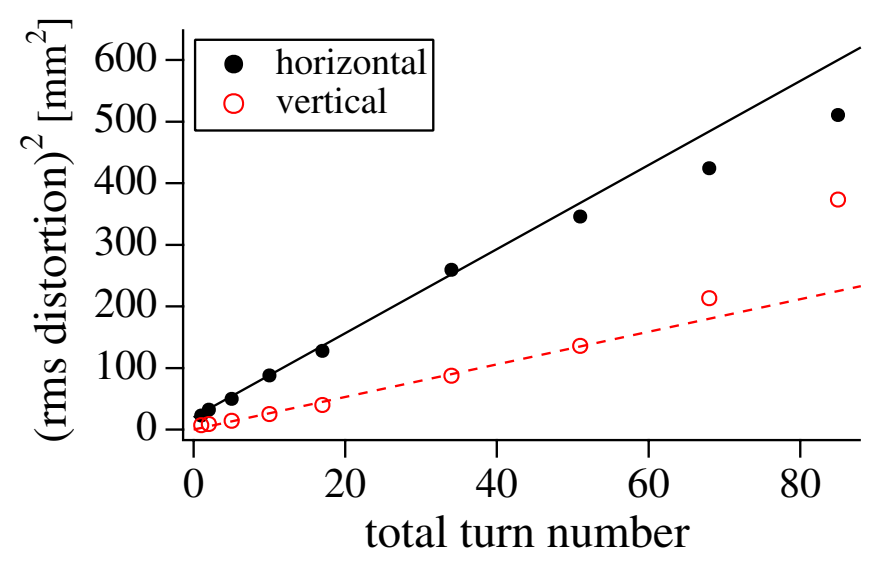

FIG. 13. (Color) Square of rms distortion for different acceleration rate. Filled and empty circles are simulation results and the solid and dashed lines are the random walk model.

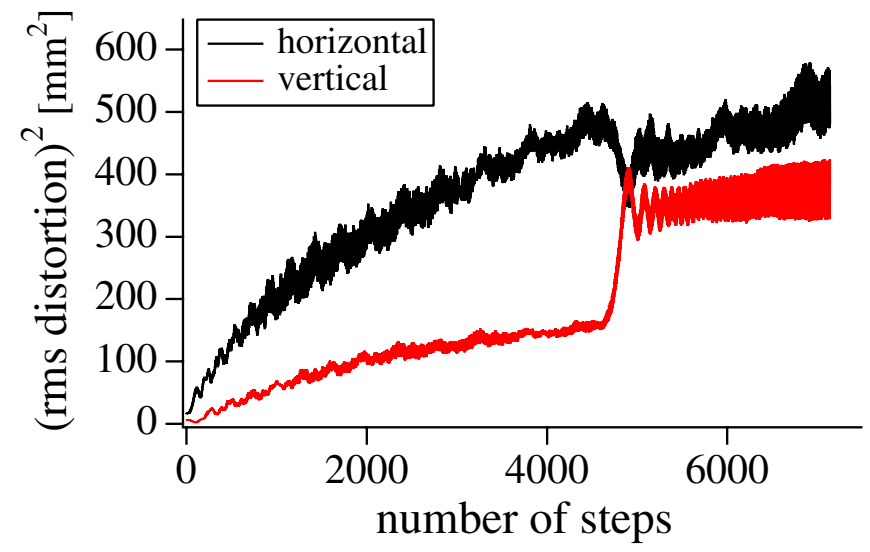

FIG. 14. (Color) Evolution of the square of horizontal and vertical rms orbit distortion when acceleration rate is 5 times slower. Exchange of distortion occurs when a particle crosses $q x-2 q y=0$ resonance.

exchange, which is a structure resonance and can be excited without any errors. Possible nonlinearities are the kinematic terms and the fringe fields.

\section{Optics distortion}

Similar analysis can be performed for optics distortion. We have calculated rms optics distortion over 501 patterns with fixed rms gradient errors of $0.1 \%$. Figure 15 shows the squared value of the rms distortion defined as

$$
(\text { rms distortion })^{2}=\left\langle\left(\sqrt{\frac{A_{\max }}{A_{0}}}-1\right)^{2}\right\rangle .
$$

If the source of the distortion is random quadrupole kicks and its strength decreases inversely proportional to the momentum, the curve should follow the dependence of $\frac{t}{1+t}$. The agreement between the simulation result and the model is quite good.
Dependence on acceleration rate, however, was not what we had expected as shown in Fig. 16. The optics distortion grows more quickly than a linear dependence on the total turn number would predict. As shown in Fig. 17, distortion as a function of number of steps no longer follows the

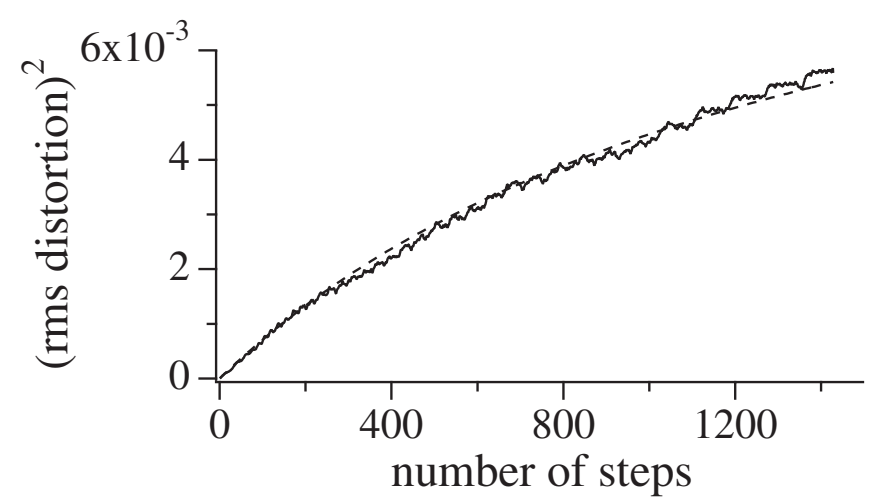

FIG. 15. Evolution of the square of rms optics distortion. There are $1428(=84$ cells $\times 17$ turns $)$ steps. The solid curve is simulation results and the dashed curve is the random walk model.

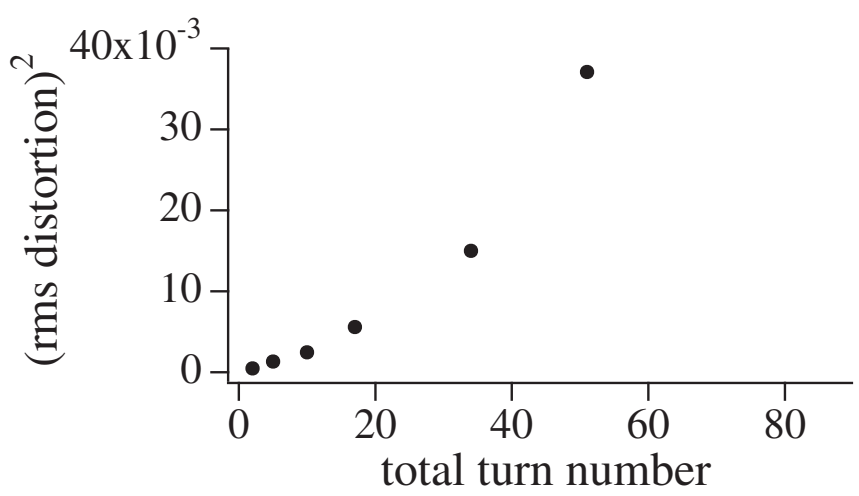

FIG. 16. Square of rms distortion for different acceleration rate.

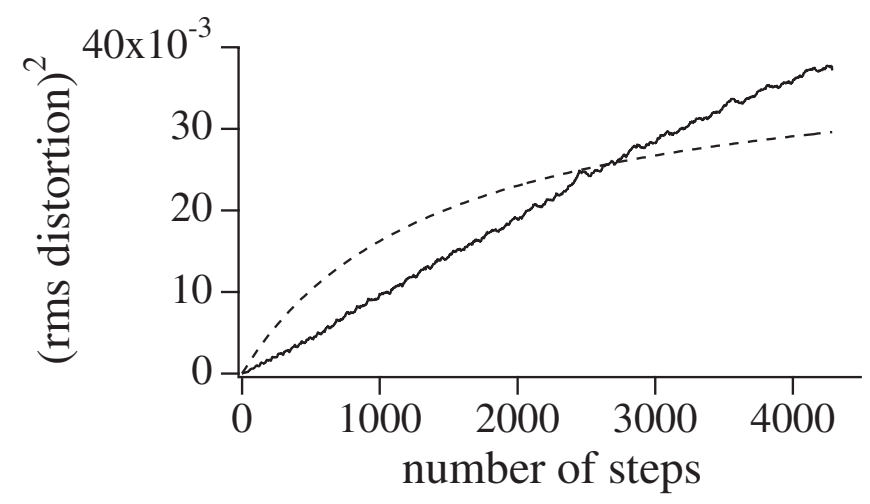

FIG. 17. Evolution of the square of rms optics distortion. There are $4284(=84$ cells $\times 51$ turns $)$ steps. The solid curve is simulation results and the dashed curve is the random walk model. 
model when the acceleration is 3 times slower than the nominal case. This suggests that the random walk model on the optics distortion may be applicable only to a certain regime of acceleration rate.

\section{Correction of distortion}

Since the distortion is not due to a resonance, it is impossible to correct the distortion with additional corrector magnets that rely on a phase relation between the magnets and the correctors at a particular betatron tune. In order to demonstrate it, we first tried to correct the vertical closed orbit distortion (COD) at several fixed momenta to check if it was effective for the case with acceleration. With 16 correctors, the vertical COD in the momentum range of 10 to $10.3 \mathrm{GeV} / c$ could be fairly suppressed as shown in Fig. 18(a). Individual corrector strength was determined iteratively to minimize the overall COD in the momentum range.

Orbit distortion of a particle with acceleration, however, does not show any improvement with those correctors as shown in Fig. 18(b). To be more quantitative, we defined an improvement factor as the ratio of rms orbit distortion before and after the additional correctors. If it is zero, the correctors correct the distortion perfectly; if it is one, there is no improvement; and if it is more than one, the correctors make the orbit distortion worse. The improvement factor becomes 0.98 in this example. We further calculated the improvement factor for other patterns of errors. The average improvement factor of 30 different lattice configurations becomes 1.04. This indicates the COD correction is indeed not effective.

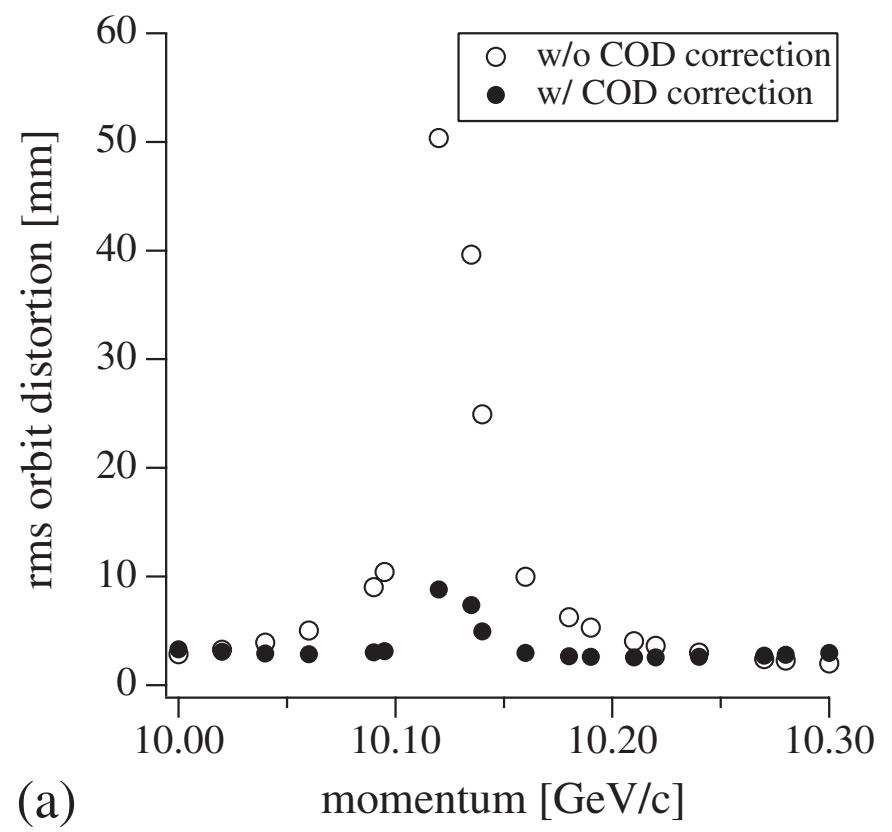

The most we can do is to measure beam positions at a certain momentum, infer alignment errors at each magnet, and move the magnets to eliminate the measured misalignment. For the optics distortion, we should measure lattice functions and correct each quadrupole strength either by an individually controlled power supply or trim coils embedded in the magnets to eliminate the source of gradient errors.

\section{SUMMARY}

By tracking simulation, we studied the orbit and optics distortion in a nonscaling FFAG with alignment and gradient errors. Although integer and half-integer resonance crossing was a primary concern because the betatron tune changes a lot during acceleration, the tracking results do not show any excitation by resonances in orbit and optics distortion at the nominal acceleration rate. The orbit and optics distortion has a linear dependence on the magnitude of errors in a ring. Rather, the simulation results indicate that the distortion is excited by random dipole and quadrupole kicks.

We applied the random walk model to analyze the simulation results. The model, however, has to be modified to take into account the time dependence of the kick strength because a particle is accelerated and kick angle decreases accordingly while the error fields are constant. As a result, the squared value of rms distortion should show a time dependence of $\frac{t}{1+t}$, where $t=0$ at injection and linearly increases to 1 at extraction. Simulation results of the rms orbit and optics distortion show a good agreement with this model.

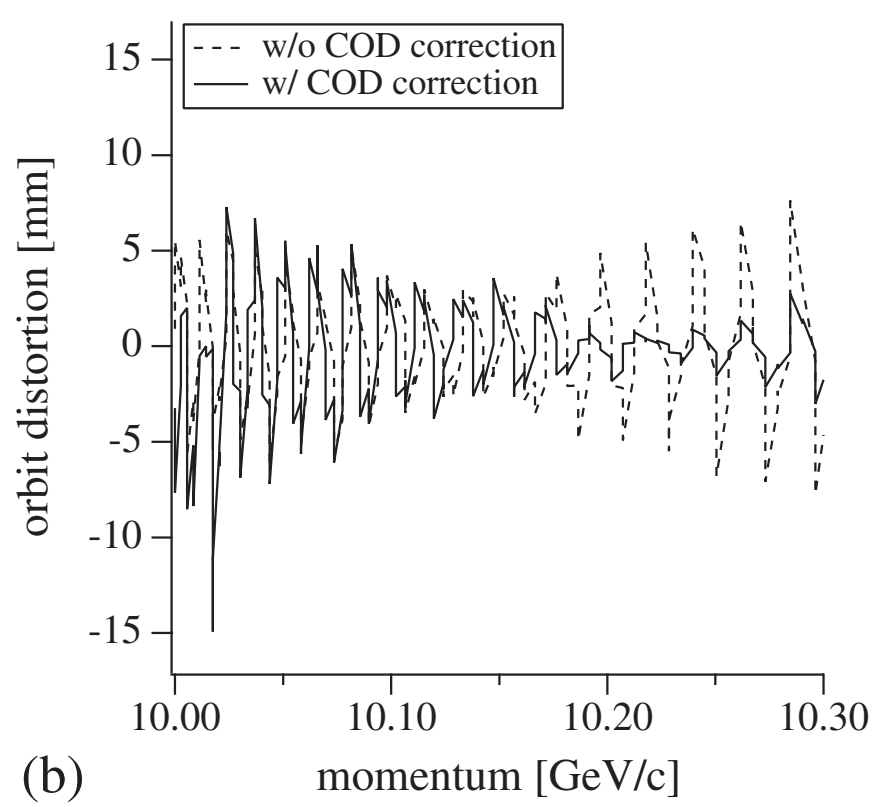

FIG. 18. (a) The rms closed orbit distortion with and without COD correction. (b) Orbit distortion of an accelerating particle with and without COD correction. 


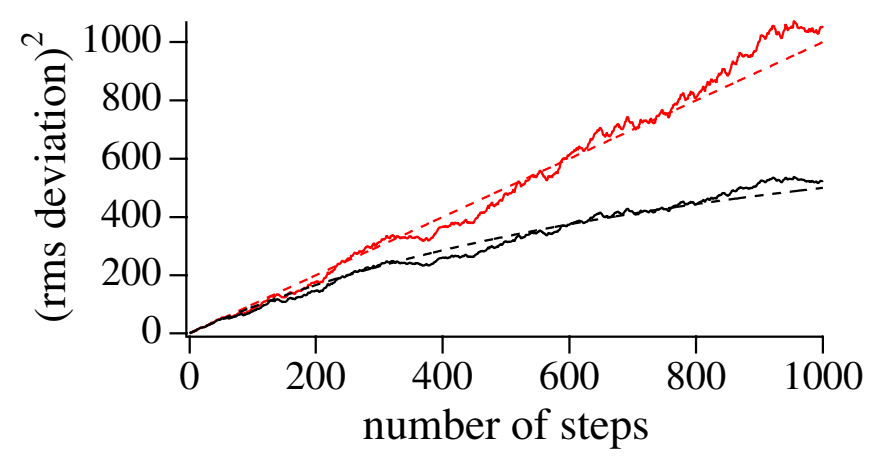

FIG. 19. (Color) The red curves show the case with the constant kick strength throughout the process and the black curves show the case with the kick strength decreasing as $\frac{1}{1+t}$. The solid curves are simulation results and the dashed curves are the model of random walk.

When the emittance is small, optics distortion causes just beam size growth due to tumbling, not necessarily emittance growth. For large muon emittance such as $30 \pi \mathrm{mm} \mathrm{rad}$, however, emittance ellipse is distorted due to nonlinearity. Since it seems impossible to correct the distortion by additional correction elements, the only way to reduce the distortion is to eliminate error sources themselves.

\section{ACKNOWLEDGMENTS}

The work is supported by the U.K. Neutrino Factory/ Particle Physics and Astronomy Research Council (PPARC) under Contract No. 2054. We would like to thank Dr. C. Prior, Dr. G. Rees, and Dr. S. Ohnuma for helpful comments on the work and the manuscript.

\section{APPENDIX}

The random walk model predicts that the rms deviation evolves with a square root dependence on time steps. In a nonscaling FFAG, the magnitude of a kick angle decreases since a beam is accelerated and orbits change although dipole and quadrupole error fields are constant. The change in kick angle is strongly dependent on acceleration so that we can modify the random walk model suitable for the study. We assume that the magnitude of a kick angle decreases as $\frac{1}{1+t}$ where $t$ is normalized time from 0 to 1 . This means that the beam momentum becomes twice as much at the end and the increase is linear with time.
Figure 19 plots the squared values of rms deviations when 100 particles go through the random walk process in 1000 time steps. In each time step, 100 particles received random kicks whose rms strength is 1 at the beginning in one-dimensional space. There are two solid curves of red and black. The red one shows the case with the constant kick strength throughout the process and the black one shows the case with the kick strength decreasing as $\frac{1}{1+t}$.

It is clear that the solid red curve increases linearly with time step (time_step) as expected. The dashed red curve is the prediction. The solid black curve, on the other hand, can be fitted by the function of (time_step $\times \frac{t}{1+t}$ ), which is the dashed black curve. With more than 1000 particles, the solid and dashed curves become indistinguishable.

[1] S. Machida, Nucl. Instrum. Methods Phys. Res., Sect. A 503, 41 (2003).

[2] K. R. Symon, D. W. Kerst, L. W. Jones, L. J. Laslett, and K. M. Terwillinger, Phys. Rev. 103, 1837 (1956).

[3] A. A. Kolomensky and A. N. Lebedev, Theory of Cyclic Accelerators (North-Holland, Amsterdam, 1966), p. 337.

[4] C. Johnstone, W. Wan, and A. Garren, Proceedings of PAC 1999, New York, p. 3068.

[5] E. Keil and A. M. Sessler, Nucl. Instrum. Methods Phys. Res., Sect. A 538, 159 (2005).

[6] D. Trbojevic, E. D. Courant, and M. Blaskiewicz, Phys. Rev. ST Accel. Beams 8, 050101 (2005).

[7] D. Trbojevic, A. G. Ruggiero, E. Keil, N. Neskovic, and A. Sessler, Proceedings of Cyclotrons 2004, Tokyo.

[8] G. H. Rees (private communication).

[9] S. Koscielniak and R. Baartman, Proceedings of PAC 2005, Knoxville, p. 3206.

[10] P. A. Sturrock, Ann. Phys. (Leipzig) 3, 113 (1958).

[11] "International Scoping Study Machine Working Group US FFAG Specifications," http://www.cap.bnl.gov/mumu/ projects/ISS/accel/us-ffag.html.

[12] C. Johnstone and S. Koscielniak, Nucl. Instrum. Methods Phys. Res., Sect. A 503, 445 (2003).

[13] S. Koscielniak and C. Johnstone, Nucl. Instrum. Methods Phys. Res., Sect. A 523, 25 (2004).

[14] S. Machida, Phys. Rev. ST Accel. Beams 9, 104002 (2006).

[15] J. S. Berg, Nucl. Instrum. Methods Phys. Res., Sect. A 570, 15 (2007).

[16] S. Y. Lee, Accelerator Physics (World Scientific, Singapore, 1999), p. 80.

[17] S. Y. Lee, Accelerator Physics (Ref. [16]), p. 93. 